\title{
$Y$ chromosome of Aisin Gioro, the imperial house of the Qing dynasty
}

\author{
Shi Yan ${ }^{1}$, Harumasa Tachibana ${ }^{2}$, Lan-Hai Wei ${ }^{1}, \mathrm{Ge} \mathrm{Yu}^{1}$, Shao-Qing Wen ${ }^{1}$ and Chuan-Chao Wang ${ }^{1}$ \\ The House of Aisin Gioro is the imperial family of the last dynasty in Chinese history-Qing dynasty (1644-1911). The Aisin \\ Gioro family originated from Jurchen tribes and founded the Manchu people before they conquered China. By investigating the \\ Y chromosomal short tandem repeats (STRs) of seven modern male individuals who claim to belong to the Aisin Gioro family \\ (three of which have full records of pedigree), we found that three of them (two of which having full pedigree, whose most \\ recent common ancestor is Nurgaci) showed very close relationship (1-2 steps of differences in 17 STRs) and possessed a \\ rare haplotype. We therefore conclude that this haplotype is the $Y$ chromosome of the House of Aisin Gioro. Further tests of \\ single-nucleotide polymorphisms indicate that they belong to haplogroup C3b2b1*-M401(xF5483), although their Y-STR \\ results indicate that they are not a part of the 'star cluster' (once linked to Genghis Khan), which belongs to the same \\ haplogroup. This study forms the base for the pedigree research of the imperial family of Qing dynasty by means \\ of genetics.
}

Journal of Human Genetics (2015) 60, 295-298; doi:10.1038/jhg.2015.28; published online 2 April 2015

\section{INTRODUCTION}

The non-recombining region of male-specific $\mathrm{Y}$ chromosome is strictly passed on from father to son. Random mutations may occur during its inheritance, thereby allowing the reconstruction of paternal phylogeny through comparison of the sequence of males' Y chromosomes. The males sharing closer common ancestor have more similar sequences, and vice versa. Commonly used markers for tracing $\mathrm{Y}$ chromosomal phylogeny include single-nucleotide polymorphisms (SNPs) and short tandem repeats (STRs). The upstream SNPs can be used for tracing relatively old (usually more than thousands of years) clades. On the other hand, using high-throughput sequencing of long regions of $\mathrm{Y}$ chromosome, we can discover rare and recent mutations, and calculate the divergence time with a theoretical highest precision of $\sim 100$ years, that is, 3-4 generations. ${ }^{1}$ The mutation rate of STRs is higher and STR recurrent mutations occur frequently, therefore relatively recent relationships can be traced by comparing STRs. $^{2}$

As both surname inheritance and genealogical records in most East Asian populations follow the paternal lineage, we can verify the precision of genealogy records using $\mathrm{Y}$ chromosomal data. In the following cases, different types of $\mathrm{Y}$ chromosomes occur in one paternal family: illegitimate son, adoption from another family and when a son inherited his mother's surname. Adoption from inside a paternal family usually cannot be recognized through analysis of $\mathrm{Y}$ chromosome. Also, it commonly happens that a family traces their genealogy to an unrelated family with the same surname, to enhance their own prestige.

Owing to the uncertainty of father-son relationship, technically, the Y chromosomal result of a man shows only the haplotype of himself rather than that of his whole paternal family. However, corresponding results of two modern individuals that were supposed as related would verify the lineages from their most recent common ancestor to both the individuals, and more tested results would make the conclusion more reliable.

Currently, the only reported Y chromosome result of historical Chinese imperial houses is that of Cáo Wèi dynasty (220-265 AD), belonging to the haplogroup $\mathrm{O} 2^{*}-\mathrm{M} 268$. This conclusion was first achieved via comparison and statistics of the $\mathrm{Y}$ chromosomes of descendants recorded in the genealogy books, then verified using the DNA extracted from the remains of Cáo Dǐng (曹鼎), Cáo Cào (曹操)'s grandfather's brother. ${ }^{3,4}$ The Y chromosomal type of Genghis Khan was once supposed to belong to the 'star cluster' under $\mathrm{C}^{*}-\mathrm{M} 217(\mathrm{xM} 48),{ }^{5}$ however, this was only deduced based on the Mongolian STR cluster with high frequency without genealogical support, and is still under debate. ${ }^{6}$

The House of Aisin Gioro (愛新覺羅, all the Manchurian names in this article apply to the Latin transcription invented by von Möllendorff ${ }^{7}$ ) originated from Jurchen tribes, and consolidated Jurchen and Mongolian tribes as well as surrounding Han Chinese into the Manju (commonly Manchu) people, and finally founded the last imperial dynasty of China - the Qing dynasty (1644-1911). Only

${ }^{1}$ Ministry of Education Key Laboratory of Contemporary Anthropology and State Key Laboratory of Genetic Engineering, Collaborative Innovation Center for Genetics and Development, School of Life Sciences, Fudan University, Shanghai, China and ${ }^{2}$ Pen name. Independent scholar, Xishiku Str. 26, Beijing 100034, China

Correspondence: Dr S Yan, Ministry of Education Key Laboratory of Contemporary Anthropology and State Key Laboratory of Genetic Engineering, Collaborative Innovation Center for Genetics and Development, School of Life Sciences, Fudan University, Songhu Road 2005, Biological Building A604, Shanghai 200438, China.

E-mail: yanshi@fudan.edu.cn

Received 19 December 2014; revised 17 February 2015; accepted 22 February 2015; published online 2 April 2015 
one study has speculated the Y chromosomal type of the House of Aisin Gioro: Xue et al. ${ }^{8}$ revealed an STR cluster under haplogroup C3c-M 48 , which includes $\sim 3.3 \%$ of males in some populations of Northeast China as well as in Mongolians. They named it as the 'Manchu cluster', and calculated its divergence time at $590 \pm 340$ years. The authors claimed that due to its recency, the expansion of this lineage should be an event recorded in history. Because of its large and widespread population, the only explanation for this cluster should be that it were the $\mathrm{Y}$ chromosome of Aisin Gioro, with the most recent common ancestor being Giocangga (覺昌安), the grandfather of Nurgaci (努爾哈赤, commonly Nurhaci, 1559-1626, the first emperor of Later Jinn (後金) dynasty). However, the authors did not provide any genealogical evidences in support of this interpretation. In addition, the figure in this report showed that the 'Manchu cluster' occurred at clearly higher frequency in Oroqen, Ewenki, Hezhe and Inner and Outer Mongolians than in Manchu and Sibe (the closest ethnic group to Manchu that separated from Manchu in early Qing dynasty). Therefore, the conclusion of this study need to be verified by further genealogical studies.

In Manchurian language, the term 'hala' (哈拉) is the surname of people who are said to have the same paternal common ancestor, although it was also suggested that people from one hala only belonged to the same tribe rather than having the same paternal origin. ${ }^{9}$ The Gioro hala (覺羅哈拉) includes sub-surnames like Aisin Gioro, Irgen Gioro (依爾根覺羅), Šušu Gioro (舒舒覺羅) and so on, with 'aisin' meaning 'gold' in Manchurian. Aisin Gioro includes the people with the same traceable ancestor with Nurgaci, that is the descendants of his great-grandfather Fuman (福滿). Since 1636, the descendants of Taksi (塔克世, father of Nurgaci) were nominated as 'uksun' (宗室, 'the imperial house'), whereas the peripheral members in Aisin Gioro were called 'gioro' (覺羅), which has a different definition from Gioro hala. After Manchu's conquest of the entirety of China, the descendants of Kangxi Emperor (great-grandson of Nurgaci and son of Fulin, 1654-1722) were called 'near uksun' (近支宗室), whereas the other uksun were called 'far uksun' (遠支宗室).

In a statistics in 1915, there were 27884 males of Aisin Gioro alive, including 16454 uksun and 11430 gioro. ${ }^{10}$ Considering natural population growth, as well as wars, political events and the policy of family planning in the recent 100 years, the currently estimated number of Aisin Gioro males is $<30000$. Owing to a decree in the Qing dynasty that $u k s u n$ were forbidden to leave the capitals-Beijing and Mukden (present Shenyang), great number of present Aisin Gioro still live in these two cities.

To understand the origin of Aisin Gioro family, their current state, as well as to review some historical rumors about the family, we investigated Aisin Gioro's Y chromosome by testing SNPs and STRs.

\section{MATERIALS AND METHODS}

We collected blood or oral samples from seven males whose families lived for generations in Beijing or Liaoning, who all claimed to belong to the House of Aisin Gioro but separated from each other more than five generations ago. The study was under the approval of the Ethics Committee of Biological Research at Fudan University, and all the samples were collected with the informed consent. Among the seven males, three have complete genealogical records inside the book of 'Genealogy of Aisin Gioro',11 whereas the others could only offer partial ancestral information. All the three with full lineage were 'far uksun', and their family lived in Beijing for generations, with two of them (samples A and D) being descendants of Dodo (多鐸, the 15th son of Nurgaci), and the other (sample B) from the House of Prince of Sù (肅親王), descendant of Hooge (豪格, the first son of the second Qing Emperor Hung Taiji 皇太極). In addition, one testee from Beijing (sample E) claimed to be from the family of Prince of Zhèng (鄭親王, descendant of Jirgalang (濟爾哈朗, nephew of Nurgaci)). A further testee (sample C) from Beijing claimed to be a descendant of 'gioro Langkio' (覺羅郎球), who is possibly the great-grandson of Soocangga (索長阿, the third son of Fuman). The other two testees are from Liaoning Province, one from Jinzhou (錦州) (sample F) also claimed to be a descendant of Dodo, and the other (sample G, without knowing his ancestors' names) from Benxi (本溪). To avoid troubles or loss of reputations of the testees and their close relatives, we do not reveal the testees' names or the information of their near ancestry.

We extracted the DNA of all these samples and amplified 17 Y-STRs (DYS19, DYS389I/II, DYS390, DYS391, DYS392, DYS393, DYS437, DYS438, DYS439, DYS448, DYS456, DYS458, DYS635, Y-GATA H4 and DYS385a/b) using Y-Filer kit (Life Technologies, CA, USA). For those samples that 17 STRs fell into the range of haplogroup C, we genotyped seven SNPs using Sanger sequencing-M217, F1396, F3535, F3273, F5483/SK1074, M546 and M401-to determine their fine phylogeny (see Table 1). STR network of C3 samples from this study and compared samples (see Results) were drawn using Network 4.6.1.2 (Fluxus, Suffolk, UK) with median joining algorithm. ${ }^{12}$

\section{RESULTS}

Among the 17 STR results of all the seven testees, three (A-a descendant of Dodo, B-a descendant of Hooge and C-a descendant of Langkio by oral history, see Figure 1) showed similar results, having STR haplotypes with only 1-2 steps of mutations among each other (Table 2 and Figure 2), whereas the others had at least six steps to them and among each other, which excludes the possibility that they share a common ancestor inside 500 years. By searching published literatures, we found two nearest samples that have two steps of difference with sample A-one is a Manchu from Xinbin (新賓) County of Liaoning Province (Ht188), ${ }^{13}$ the other is from northeastern part of Mongolia (MG3_5). ${ }^{14}$ We also searched the largest Y-STR data set-http://www.ysearch.org, but could not find any samples as close as two steps.

The phylogeny of these samples were tested for seven SNPs (Table 1 and Figure 3), among which M217 (haplogroup C3) was well known in numerous literatures; ${ }^{14-16}$ F1396, F3535 and F3273/M504 were

Table 1 Tested SNPs

\begin{tabular}{|c|c|c|c|c|}
\hline SNP & Position (hg19) & Mutation & Forward primer (5'-3') & Reverse primer ( $\left.5^{\prime}-3^{\prime}\right)$ \\
\hline M217 & 15437333 & $A>C$ & CCAGTATCTCCAAAATCCTCTCGTA & CGTAAGCATTTGATAAAGCTGCTGT \\
\hline F1396 & 8746611 & $\mathrm{C}>\mathrm{T}$ & CACACСССТCTTGTGTCACAGCA & ACCTGCCTGTGTGCAGTCTTTCTA \\
\hline F3535 & 23768635 & $\mathrm{G}>\mathrm{T}$ & TCACCAGGTTGTATCCTCACCTGT & AGAACCACACAGCCTCATGAGCTG \\
\hline F3273/M504 & 21888793 & $\mathrm{~T}>\mathrm{C}$ & CTTTTGATAAAAGTATCATGTGTTCAGT & CACAATGTAGTTGAAAATTGAAGTTGTA \\
\hline M546 & 19060966 & $\mathrm{C}>\mathrm{A}$ & CCTTGTAAAAGCCAAGCTGCСCTT & TTAGGTTTTACAATAGTGATGCTATTCT \\
\hline M401 & 2745569 & TG ins & GCTGGTCTCAAACTTCTGACCTTG & GCTGGTCTCAAACTTCTGACCTTG \\
\hline F5483/SK1074 & 19086774 & $\mathrm{~T}>\mathrm{A}$ & AAAGTCTGCCATTATGATGATATAGTTC & CTTCAAGTATACGTGAAGGCTAAATG \\
\hline
\end{tabular}




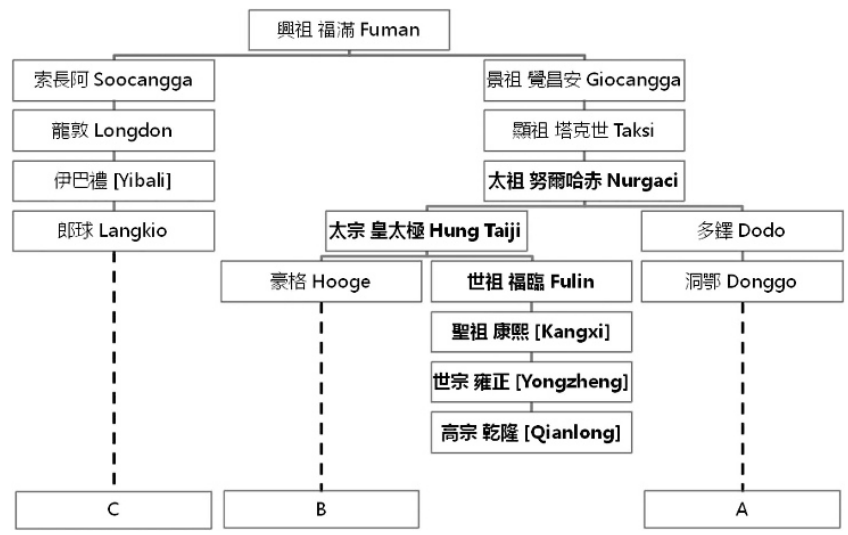

Figure 1 The genealogy of the three samples $(A-C)$ that have close STR results. The genealogy of $a$ and $b$ is fully described, whereas $c$ has only oral history. Those labeled in bold are emperors of Later Jin and Qinng dynasty. The names are in Manchurian transcription, except that those in Chinese pinyin are labeled in square brackets. Given the definition of Fuman as the first generation and Nurgaci in the fourth generation, A and B are in the seventeenth and sixteenth generation, respectively. Generation order of sample $\mathrm{C}$ is unclear.

discovered from high-throughput sequencing of Y chromosomes of Mongolian and Manchu samples; ${ }^{17}$ M401 and M546 were found in Mongolian and many related populations, especially the 'star cluster', which was suspected to be the clan of Genghis Khan, which also belongs to the clade C3b2b1-M401; 14 and F5483/SK1074 was discovered from Orochon and Daur samples. ${ }^{18}$ The samples A, B and C were genotyped as belonging to haplogroup C3b2b1*-M401(xF5483), the same as the 'star cluster', despite 10 steps of difference in 17 STRs between sample B and the central value of the 'star cluster' (Table 2).

\section{DISCUSSION}

Although there is large confidence interval for the divergence time estimated using Y-STRs and ongoing debate about the STR mutation rate (from the 'effective mutation rate' of $6.9 \times 10^{-4}$ in 25 years, that is, one mutation per 36231 years, ${ }^{19}$ to the father-to-son mutation rate of $3 \times 10^{-3}$ per generation, ${ }^{20}$ that is, one mutation per 10000 years), the calculated age can give a rough image about the divergence time. Using the software network with a medium mutation rate of one mutation in 20000 years for 15 STRs with exceptions of DYS385a/b (as DYS385 loci may be converted from each other and cause complexity in time estimation ${ }^{21}$ ), we estimated the most recent ancestor of A, B and C as $666 \pm 471$ years before present. This time is in accordance with the genealogy (Fuman as the most recent common ancestor, $\sim 500$ years before present). Adding the Xinbin Manchu sample Ht188, the calculated divergence time became $1333 \pm 653$, which is much older than Aisin Gioro's time depth. Therefore, we suggest that the Y-STR combination of the three samples represent that of Aisin Gioro family.

This Y-STR haplotype is rare in modern populations, including Han, Manchu and Mongol, and we found the samples nearer than two steps to sample B are only from Aisin Gioro family, therefore a member in question with less than two steps to this haplotype has a great chance that he really belongs to Aisin Gioro. Xinbin County in Liaoning is the location of Hetu Ala (赫圖阿拉) city where Nurgaci arose, and there can be certain amount of Aisin Gioro's relatives (with divergence time of 500 to 1000 years). However, we found only one sample (Ht188) in 231 published Manchu samples from Xinbin that

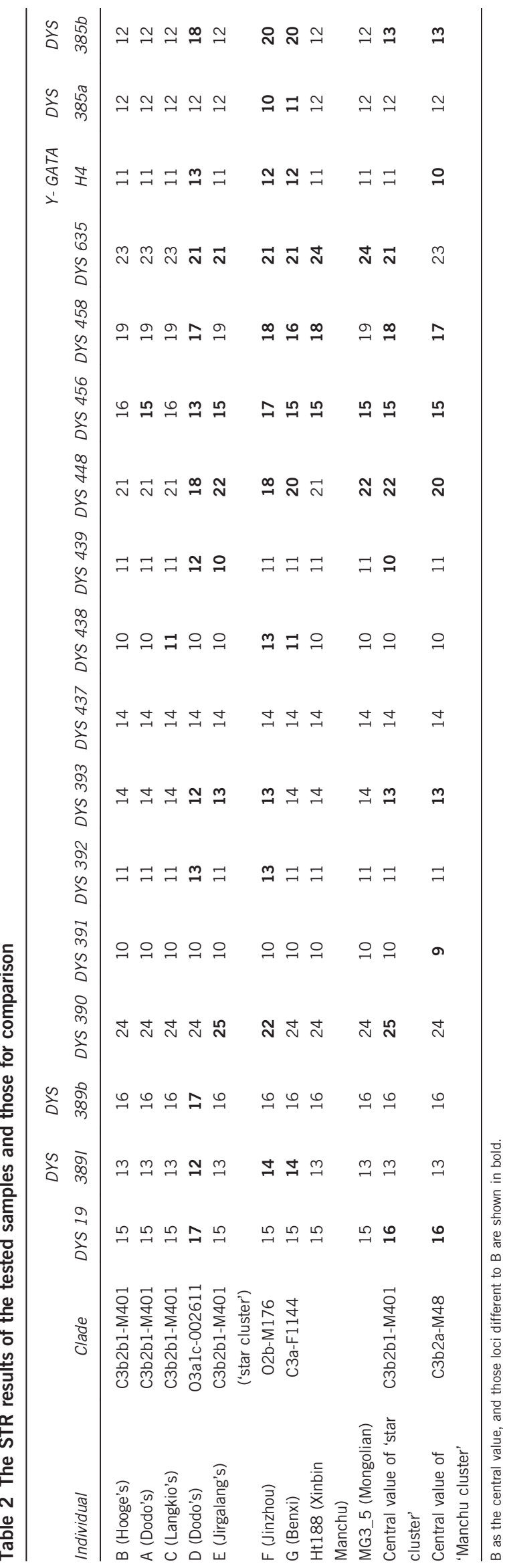




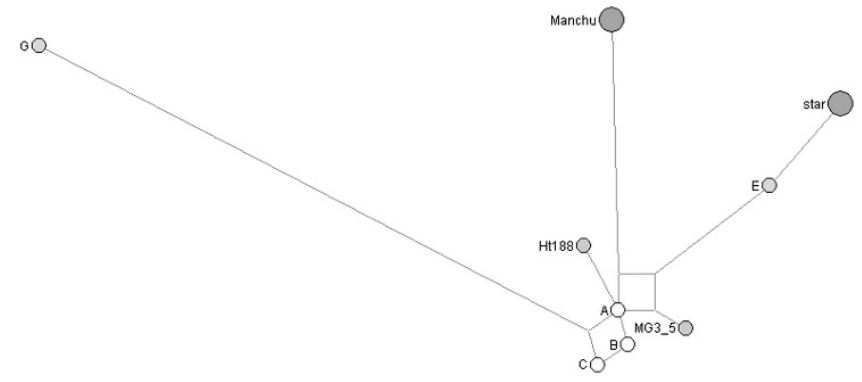

Figure 2 Network of C3-M217 samples from this study (yellow for concluded Aisin Gioro and green for two others, E and G), as well as two samples (Ht188 and MG3_5, in gray) and the center value of 'star cluster' and 'Manchu cluster') for comparison. A full color version of this figure is available at the Journal of Human Genetics journal online.

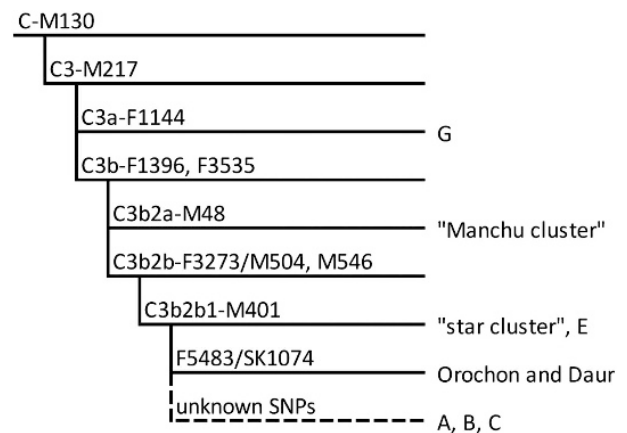

Figure 3 Phylogenetic tree of haplogroup C concerning the single-nucleotide polymorphisms genotyped in this study.

have two steps of STR difference to sample A, which means that the Aisin Gioro family is not frequent in a normal Manchu population. However, we still expect to find more individuals with this haplotype from Manchu in Beijing and Shenyang. This study also shows that, among those who claim to be Aisin Gioro without a clear genealogy, the chance that one really belongs to this lineage (in biological sense) is not high. In contrast to the conjecture that Aisin Gioro belongs to C3b2a-M48 'Manchu cluster' based on frequency data, this study showed that the common imagination that a powerful emperor or king's descendants should comprise a great proportion of a population can be improper.

Understanding of Y chromosomal type of Aisin Gioro will benefit historical research on Jurchen, Manchu and the Qing dynasty, and will help to confirm or disprove some rumors of genealogical relationships. For example, some scholars inside Aisin Gioro claim that the Gioro hala were descendants of the Hū Zōng Emperor of Sòng dynasty (宋徽宗, 1082-1135), who was captured by the Jurchen Jin dynasty and exiled to Northeast China. ${ }^{9}$ There is also a rumor that the Qianlong Emperor was not the biological son of the Yongzheng Emperor (son of Kangxi Emperor). Although no descendants of Qianlong were included in this study, this rumor can be easily examined in the future.

It should be noted that during the Chinese history, the father-son relationship in social law is not equal to that in biological sense. Many adoption events owing to sonlessness were recorded in the genealogy of Aisin Gioro; most of the adoption events occurred between closely related clans, although adoption from other surnames also happened. Therefore, it is not proper to judge social family heredity simply using the biological evidence.

\section{CONFLICT OF INTEREST}

The authors declare no conflict of interest.

\section{ACKNOWLEDGEMENTS}

We are grateful for the trust of the sample donors. This research is supported by the grants from the National Science Foundation of China (31271338), and from Ministry of Education (311016).

1 Xue, Y. L., Wang, Q. J., Long, Q., Ng, B. L., Swerdlow, H. \& Burton, J. et al. Human Y chromosome base-substitution mutation rate measured by direct sequencing in a deeprooting pedigree. Curr. Biol. 19, 1453-1457 (2009).

2 Ge, J. Y., Budowle, B., Aranda, X. G., Planz, J. V., Eisenberg, A. J. \& Chakraborty, R. Mutation rates at $Y$ chromosome short tandem repeats in Texas populations. Forensic Sci. Int.-Gen. 3, 179-184 (2009).

3 Wang, C., Yan, S., Hou, Z., Fu, W., Xiong, M. \& Han, S. et al. Present Y chromosomes reveal the ancestry of Emperor CAO Cao of 1800 years ago. J. Hum. Genet. 57, 216-218 (2012).

4 Wang, C.-C., Yan, S., Yao, C., Huang, X.-Y., Ao, X. \& Wang, Z. et al. Ancient DNA of emperor CAO Cao's granduncle matches those of his present descendants: a commentary on present $Y$ chromosomes reveal the ancestry of Emperor CAO Cao of 1800 years ago. J. Hum. Genet. 58, 238-239 (2013).

5 Zerjal, T., Xue, Y. L., Bertorelle, G., Wells, R. S., Bao, W. D. \& Zhu, S. L. et al. The genetic legacy of the Mongols. Am. J. Hum. Genet. 72, 717-721 (2003).

6 Abilev, S., Malyarchuk, B., Derenko, M. Wozniak, M., Grzybowski, T. \& Zakharov, I. The Y-chromosome C3*star-cluster attributed to Genghis Khan's descendants is present at high frequency in the Kerey Clan from Kazakhstan. Hum. Biol. 84, 79-89 (2012).

7 von Möllendorff, P. G. A Manchu Grammar (American Presbyterian Mission Press, Shanghai, 1892).

8 Xue, Y. L., Zerjal, T., Bao, W. D., Zhu, S. L., Lim, S. K. \& Shu, Q. F. et al. Recent spread of a Y-chromosomal lineage in northern China and Mongolia. Am. J. Hum. Genet. 77, 1112-1116 (2005).

9 Jin, G. P., Jin, Q. Z. \& Ula, X. C. Collection of Works of Three Generations of Aisin Gioro (Yuanfang Press, 1996).

10 Qu, D. Y. Polygyny system and population question of imperial house of Qing Dynasty. Res. Manchuriol. 1, 26-54 (1992).

11 Aisin Gioro, C. L. Genealogy of Aisin Gioro (Xueyuan Press, 1998).

12 Bandelt, H. J., Forster, P. \& Rohl, A. Median-joining networks for inferring intraspecific phylogenies. Mol. Biol. Evol. 16, 37-48 (1999).

$13 \mathrm{He}, \mathrm{J}$. \& Guo, F. Population genetics of 17 Y-STR loci in Chinese Manchu population from Liaoning province, Northeast China. Forensic Sci. Int.-Gen. 7, E84-E85 (2013).

14 Di Cristofaro, J., Pennarun, E., Mazieres, S., Myres, N. M., Lin, A. A. \& Temori, S. A et al. Afghan Hindu Kush: where Eurasian sub-continent gene flows converge. PLoS ONE 8, e76748 (2013).

15 Xue, Y. L., Zejal, T., Bao, W. D., Zhu, S. L., Shu, Q. F. \& Xu, J. J. et al. Male demography in East Asia: a north-south contrast in human population expansion times. Genetics 172, 2431-2439 (2006).

16 Zhong, H., Shi, H., Qi, X. B., Xiao, C. J., Jin, L. \& Ma, R. L. Z. et al. Global distribution of $Y$-chromosome haplogroup $C$ reveals the prehistoric migration routes of African exodus and early settlement in East Asia. J. Hum. Genet. 55, 428-435 (2010).

17 Yan, S., Wang, C.-C., Zheng, H.-X., Wang, W., Qin, Z.-D. \& Wei, L.-H. et al. Y chromosomes of $40 \%$ chinese descend from three neolithic super-grandfathers. PLoS ONE 9, e105691 (2014).

18 Lippold, S., Xu, H., Ko, A., Li, M., Renaud, G. \& Butthof, A. et al. Human paternal and maternal demographic histories: insights from high-resolution $Y$ chromosome and mtDNA sequences. Investig. Genet. 5, 1-12 (2014).

19 Zhivotovsky, L. A., Underhill, P. A., Cinnioğlu, C., Kayser, M., Morar, B. \& Kivisild, T. et al. The effective mutation rate at $Y$ chromosome short tandem repeats, with application to human population-divergence time. Am. J. Hum. Genet. 74, 50-61 (2004).

20 Heyer, E., Puymirat, J., Dieltjes, P., Bakker, E. \& deKnijff, P. Estimating Y chromosome specific microsatellite mutation frequencies using deep rooting pedigrees. Hum. Mol. Genet. 6, 799-803 (1997).

21 Balaresque, P., King, T. E., Parkin, E. J., Heyer, E., Carvalho-Silva, D. \& Kraaijenbrink T. et al. Gene conversion violates the stepwise mutation model for microsatellites in Y-chromosomal palindromic repeats. Hum. Mutat. 35, 609-617 (2014). 